This item was submitted to Loughborough's Research Repository by the author.

Items in Figshare are protected by copyright, with all rights reserved, unless otherwise indicated.

\title{
MAGLEV suspensions - a sensor optimisation framework
}

PLEASE CITE THE PUBLISHED VERSION

PUBLISHER

(C) IEEE

LICENCE

CC BY-NC-ND 4.0

\section{REPOSITORY RECORD}

Michail, Konstantinos, Argyrios C. Zolotas, Roger M. Goodall, and John T. Pearson. 2019. "MAGLEV Suspensions - a Sensor Optimisation Framework". figshare. https://hdl.handle.net/2134/3350. 
This item was submitted to Loughborough's Institutional Repository by the author and is made available under the following Creative Commons Licence conditions.

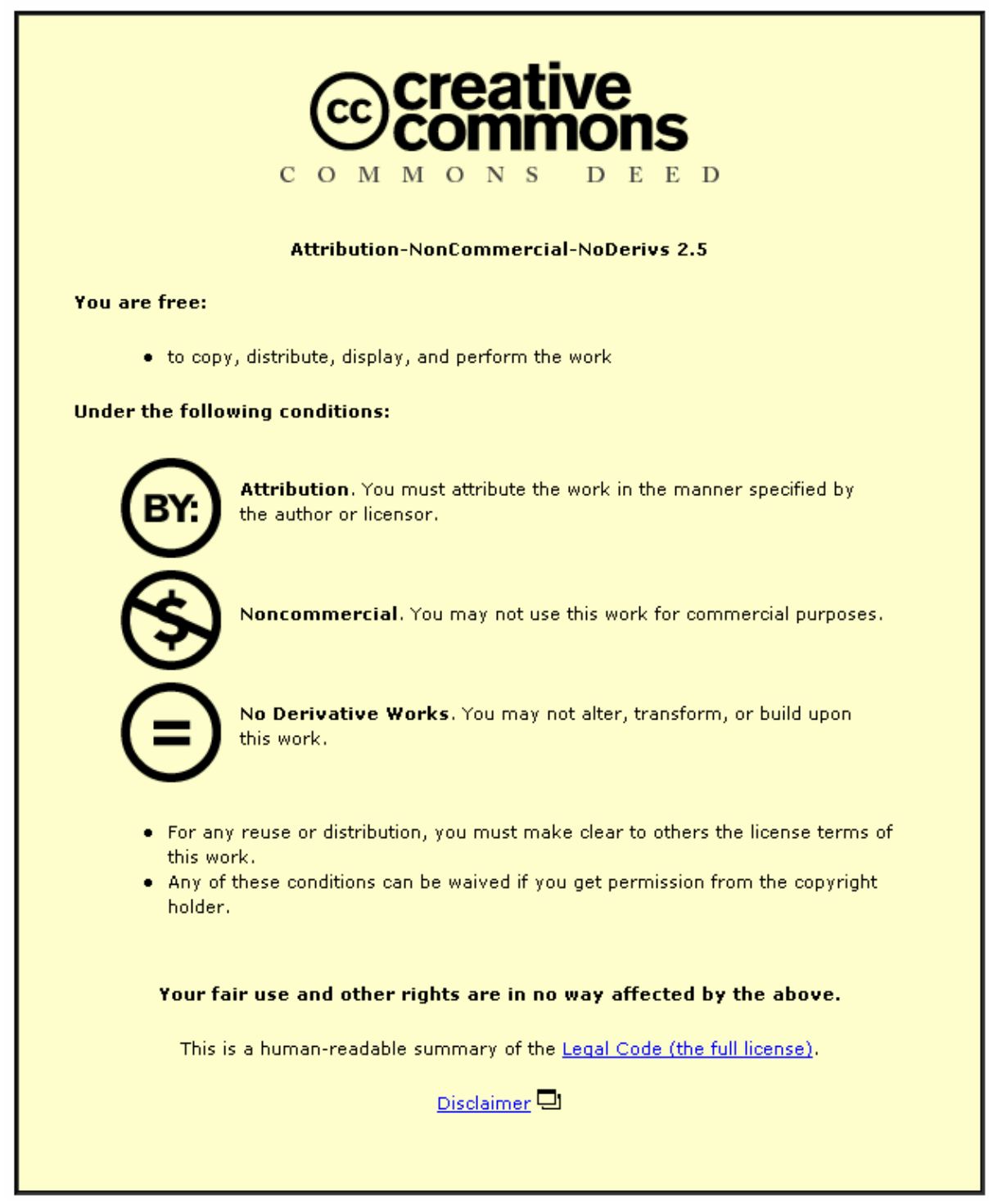

For the full text of this licence, please go to: http://creativecommons.org/licenses/by-nc-nd/2.5/ 


\title{
MAGLEV suspensions - A sensor optimisation framework
}

\author{
Konstantinos Michail Argyrios Zolotas Roger Goodall John Pearson
}

\begin{abstract}
In this paper, a systematic framework for optimised sensor configurations is implemented via $H_{\infty}$ Loop Shaping Procedure. The optimisation framework, gives the sensor sets that satisfy predefined user criteria and the preset constraints required for the MAGnetic LEVitated suspension performance via evolutionary algorithms. The scheme is assessed via appropriate simulations for its efficacy.
\end{abstract}

\section{INTRODUCTION}

During the last years, MAGnetic LEVitation (MAGLEV) systems offer a number of advantages over the conventional trains therefore is a developing area that is attractive to transport industry. Particularly, the MAGLEV train does not have mechanical contact with the rails and therefore, friction, mechanical losses, vibration and noise are reduced significantly. Two types of electromagnetic suspensions are used: The Electromagnetic suspension (EMS) that is levitated by producing an attractive force to the rail, and the Electrodynamic suspension (EDS) which is levitated via a repulsive force to the rail. A useful overview for MAGLEV technologies is given in [12].

The proposed framework utilises the loop-shaping design procedure (LSDP) of MacFarlane and Glover for the design of robust multiple input - multiple output (MIMO) systems based on shaping the open loop transfer function using a series appropriately chosen weighting functions [13]. Note that a scheme on LSDP on a MAGLEV suspension application was presented in [2], however on the control system design rather than the optimisation of sensor configurations.

In this paper, the linearised model of an EMS suspension is considered with five possible output measurements (one of which is a primary measurement, that of airgap measurement, explained in more details in the main body of the work). The LSDP method is applied for each given sensor set. To optimise each sensor set, and in particular tuning all weighting functions required is a difficult and timeconsuming task. Thus, a heuristics approach is followed, based on [5], able to search randomly in a predefined search space and find the optimum weighting functions which result to a Pareto optimal controller solution for the corresponding sensor set. In fact, evolutionary algorithms have been and are now commonly used in engineering problems and more importantly are proved to perform satisfactory in the

Authors are with Control Systems Group, Department of Electronic and Electrical Engineering, Loughborough university, UK emails: \{k.michail, a.c.zolotas, r.m.goodall, j.t.pearson\} @lboro.ac.uk

This work was supported in part under the EPSRC (UK) project Grant Ref. EP/D063965/1 and BAE Systems (SEIC),UK. case of hard engineering optimisation problems [6]. Different evolutionary algorithms are presented in [11] that are implemented in practical applications. The Non-dominated Sorting Genetic Algorithms II (NSGAII) introduced in [4] as a powerful optimisations tool, and is implemented in the proposed framework. The paper is organised as follows: Section II discusses the linearised model of the MAGLEV suspension and the input disturbances to the system. Section III presents the requirements of the suspension, the objective functions to minimise, the overall problem formulation, and the genetic algorithm parameter adjustment. Simulations and data analysis of the scheme are given in section IV with parametric uncertainty considerations in section V. Conclusions with future work are given in section VI.

\section{LineArised MAGLEV Suspension ModeL}

The diagram of a one degree-of-freedom, quarter-car electromagnetic suspension system is shown in Fig.1. The suspension consists of an electromagnet with a ferromagnetic core and a coil of $N$ turns which is attracted to the rail that is made out of ferromagnetic material. The carriage mass is attached on the electromagnet, with $z_{t}$ being the rail position and $z$ the electromagnet position. The airgap $\left(z_{t}-z\right)$ is to be maintained close to the operating condition required. The

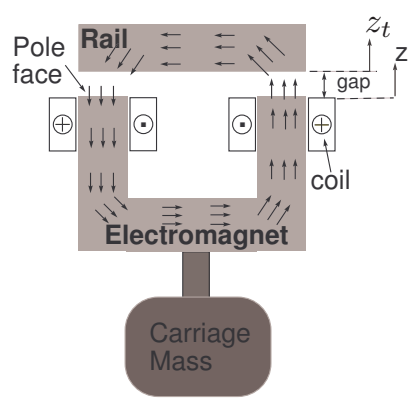

Fig. 1. Suspension system for MAGLEV

LTI state space model is derived by considering the operating point (nominal) values of the coil current $I_{0}$, flux $B_{0}$, force $F_{0}$ and airgap $G_{0}$. The following relationships hold

$$
\begin{array}{ll}
F=f+F_{0}, & B=b+B_{0} \\
G=\left(z_{t}-z\right)+G_{0}, & I=i+I_{0}
\end{array}
$$

where, $f, b,\left(z_{t}-z\right)$ and $i$ are small variations around their nominal values. The fundamental magnetic relationships are $F \propto B^{2}$ and $B \propto I / G$, thus, the linearised expressions for 
the magnet are [9]

$$
\begin{gathered}
b=K_{i} i-K_{\left(z_{t}-z\right)}\left(z_{t}-z\right) \\
f=K_{b} b
\end{gathered}
$$

where $K_{i}=B_{0} / I_{0}, K_{\left(z_{t}-z\right)}=B_{0} / G_{0}$ and $K_{b}=2 F_{0} / B_{0}$. The voltage $v$, applied to the coil is given by:

$$
v=R i+L \frac{d i}{d t}+N A \frac{d b}{d t}
$$

where $N$ is the number of coil turns, $R$ the coil resistance, $A$ is the pole face area and $L$ the coil inductance. Moreover, the force $f$ depends on the mass $M$ and the vertical acceleration $\ddot{z}$.

$$
f=M \ddot{z} \quad \text { and } \quad f=K_{b} b
$$

therefore, from (5) and (2) the equation for $\ddot{z}$ is

$$
\ddot{z}=\frac{K_{b} K_{i}}{M} i-\frac{K_{b} K_{\left(z_{t}-z\right)}}{M}\left(z_{t}-z\right)
$$

where $\left(z_{t}-z\right)$ is the airgap between the rail and the electromagnet. Also, from (2) and (6) the current equation is

$$
\frac{d i}{d t}=\frac{V}{L+N A K_{i}}+\frac{N A K_{\left(z_{t}-z\right)}}{L+N A K_{i}}\left(\dot{z}_{t}-\dot{z}\right)-\frac{R i}{L+N A K_{i}}
$$

and from (6) and (7) a state vector can be constructed as follows

$$
X=\left[\begin{array}{lll}
i & \dot{z} & \left(z_{t}-z\right)
\end{array}\right]^{T}
$$

with the relevant state space expression given by

$$
\dot{X}=A_{g} X+B_{v} v+B_{z} \dot{z}_{t}, \quad y=C X
$$

where matrices

$$
\begin{gathered}
A_{g}=\left(\begin{array}{ccc}
-\frac{R}{L+K_{i} N A} & -\frac{K_{\left(z_{t}-z\right)} N A}{L+K_{i} N A} & 0 \\
\frac{K_{b} K_{i}}{M} & 0 & -\frac{K_{b} K_{\left(z_{t}-z\right)}}{M} \\
0 & -1 & 0
\end{array}\right) \\
\left(\begin{array}{cc}
B_{v} & B_{z}
\end{array}\right)=\left(\begin{array}{ccc}
\frac{1}{L+K_{i} N A} & \frac{K_{\left(z_{t}-z\right)} N A}{L+N A K_{i}} \\
0 & 0 \\
0 & 1
\end{array}\right) \\
C=\left(\begin{array}{ccc}
1 & 0 & 0 \\
K_{i} & 0 & -K_{\left(z_{t}-z\right)} \\
0 & 0 & 1 \\
0 & 1 & 0 \\
\frac{K_{b} K_{i}}{M} & 0 & -\frac{K_{b} K_{\left(z_{t}-z\right)}}{M}
\end{array}\right)
\end{gathered}
$$

Note that the output matrix in (12) refers to all possible measurements that can be considered $\left(y=\left[\begin{array}{ll}i b\left(z_{t}-z\right) \dot{z} \ddot{z}\end{array}\right]^{T}\right)$. The parameter values for an one tone suspension system are shown in Table I. Note that 'Var' is the parameter ('Param.') percentage variation - from its nominal ('Nom.') value - that is used for the robustness analysis in section V. It is worth mentioning that the maglev system is open-loop unstable.

\section{A. Rail disturbances to the suspension}

Two track input characteristics are considered, i.e. deterministic changes such as gradients or curves and stochastic (random) changes in the track position due to misalignments.
TABLE I

MAGNETIC SUSPENSION PARAMETERS

\begin{tabular}{|c|c|c|c|c|c|}
\hline Param. & Nom. & Var.(\%) & Param. & Nom. & Var.(\%) \\
$\mathrm{M}$ & $1000 \mathrm{~kg}$ & 10 & $\mathrm{R}$ & $10 \Omega$ & 50 \\
$G_{0}$ & $0.015 \mathrm{~m}$ & - & $\mathrm{L}$ & $0.1 \mathrm{H}$ & 50 \\
$B_{0}$ & $1 T$ & 10 & $\mathrm{~N}$ & 2000 & - \\
$I_{0}$ & $10 \mathrm{~A}$ & 10 & $\mathrm{~A}$ & $0.01 \mathrm{~m}^{2}$ & - \\
$F_{0}$ & $10000 \mathrm{~N}$ & 10 & & & \\
\hline
\end{tabular}

1) Random input: Random behavior of the rail position is caused as the vehicle moves along by track-laying inaccuracies and steel rail discrepancies. Consider the vertical direction, the velocity variations are quantified by a doublesided power spectrum density (PSD) which in the frequency domain is expressed by

$$
S_{\dot{z}_{t}}=\pi A_{r} V
$$

where, $V$ is the vehicle speed (in this work is taken as $15 \mathrm{~m} / \mathrm{s}$ ) and $A_{r}$ represents the track roughness equal to $1 \times$ $10^{-7} \mathrm{~m}$ (for a typical high quality track). The corresponding (one-sided) autocorrelation function is given by

$$
R(\tau)=2 \pi^{2} A_{r} V \delta(\tau)
$$

2) Deterministic input: The main deterministic inputs to a suspension for the vertical direction are transitions onto gradients. In this work, the deterministic input components utilized are shown in Fig.2 and represent a gradient of $5 \%$ at a vehicle speed of $15 \mathrm{~m} / \mathrm{s}$ and an allowed acceleration of $0.5 \mathrm{~m} / \mathrm{s}^{2}$.

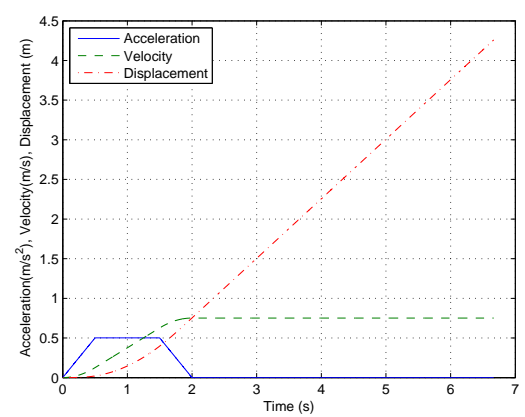

Fig. 2. Deterministic input to the suspension with a vehicle speed of $15 \mathrm{~ms}^{-1}$ and $5 \%$ gradient.

\section{SENSOR OPTIMISATION FRAMEWORK}

\section{A. MAGLEV suspension requirements}

Fundamentally there is a trade off between the deterministic and the ride quality (stochastic response) of the MAGLEV suspension. In this case, the deterministic characteristics are limited to the values shown on Table II. based on the performance requirements described in [7] and [8] for low speed MAGLEV trains. Stochastic characteristics have been set as objectives to be minimised. i.e minimise the vertical acceleration (improve ride) quality and the RMS 
current variations, as well as the $H_{\infty}$ performance $\left(\gamma_{o p t}\right)$ index.These objectives can be can be formally written as

$$
\phi_{1}=i_{r m s}, \quad \phi_{2}=\ddot{z}_{r m s}, \quad \phi_{3}=\gamma_{o p t}
$$

At this point some constraint relaxation is allowed to the vertical acceleration and the robust stability margin $(\epsilon)$. The 0.25 robust stability margin that allows $25 \%$ coprime factor uncertainty, is limited to 0.15 and the vertical acceleration to $1 \mathrm{~m} / \mathrm{s}^{2}$ (although these relaxations do not cause problems in the optimisation procedure as they are defined to the selection criteria).

TABLE II

CONSTRAINTS FOR THE MAGNETIC SUSPENSION PERFORMANCE.

\begin{tabular}{|c|c|}
\hline suspension limitations & Value \\
\hline RMS acceleration $\left(\simeq 5 \%^{\prime} g^{\prime}\right),\left(\ddot{z}_{r m s}\right)$ & $<0.5 m s^{-2}$ \\
RMS airgap variation, $\left(\left(z_{t}-z\right)_{r m s}\right)$ & $<5 \mathrm{~mm}$ \\
Maximum airgap deviation, $\left(\left(z_{t}-z\right)_{p}\right)$ & $<7.5 \mathrm{~mm}$ \\
Control effort, $\left(V_{p}\right)$ & $<300 \mathrm{~V}\left(3 \mathrm{I}_{0} R_{0}\right)$ \\
Settling time, $\left(t_{s}\right)$ & $<3 \mathrm{~s}$ \\
Air gap Steady state error, $\left(\left(z_{t}-z\right) e_{s s}\right)$ & $=0$ \\
Robust stability margin $\epsilon$ & $>0.25$ \\
\hline
\end{tabular}

\section{B. $H_{\infty}$ loop-shaping design}

The design of the optimised controller is based on the normalised coprime-factor plant description, proposed by McFarlane and Glover [13], which incorporates the simple performance/robustness tradeoff obtained in loop shaping, with the normalised Left Coprime (LCF) robust stabilization method as a means of guaranteeing closed-loop stability. The design technique forms a two stage process. The design method proceeds by shaping the open-loop characteristics of the plant by means of the weighting functions $W 1$ and $W 2$ (see Fig. 3(a)). The plant is temporarily redefined as $\hat{G}(s)=W 2 G W 1$ and the $H_{\infty}$ optimal controller $\hat{K}(s)$ is calculated. In the final stage, the weighting functions are merged with the controller by defining the overall controller $\mathrm{K}(\mathrm{s})=W 1 \hat{K} s W 2$ as shown in Fig. 3(b). The size of model

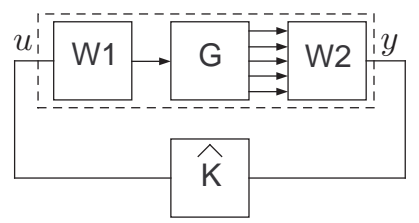

(a) Shaped plant.

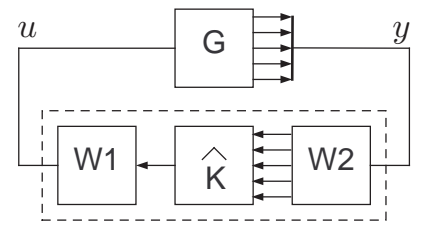

(b) Final controller.
Fig. 3. $H_{\infty}$ loop-shaping design.

uncertainty is quantified by the stability radius $\epsilon$ (refer to [13] and [14] for more details), i.e. the stability margin. For values of $\epsilon>0.25,25 \%$ coprime factor uncertainty is allowable.

We aim to keep the filter functions and thus the controller as simple as possible. Thus, the $W 1$ pre-compensator, is chosen as a single scalar weighting function set to unity. For the $W 2$ post-compensators there can be five weighting functions that are used depending on the selected sensor set. The airgap $\left(z_{t}-z\right)$ measurement is a compulsory measurement required for proper maglev control of the magnet distance from the rail and thus a low pass filter $\left(W_{\left(z_{t}-z\right)}\right)$ is chosen with integral action allowing zero steady state airgap error. The weighting functions are given as

$$
W 1=1 ; \quad W 2=\operatorname{diag}\left(W_{i}, W_{b}, W_{\left(z_{t}-z\right)}, W_{\dot{z}}, W_{\ddot{z}}\right)
$$

with,

$$
W_{\left(z_{t}-z\right)}=\left(\frac{\frac{s}{M_{p}^{1 / n_{p}}}+\omega_{b}}{s+\omega_{b} A_{p}^{1 / n_{p}}}\right)^{n_{p}}
$$

. The above results to a minimum phase and stable weighting filter with roll-off rate $n_{p}$. The filter structure for $\left(z_{t}-z\right)$ is shown in Fig. 4. Note that there exist $2^{4}$ available sensor setcombinations (recall that the airgap sensor is always required).

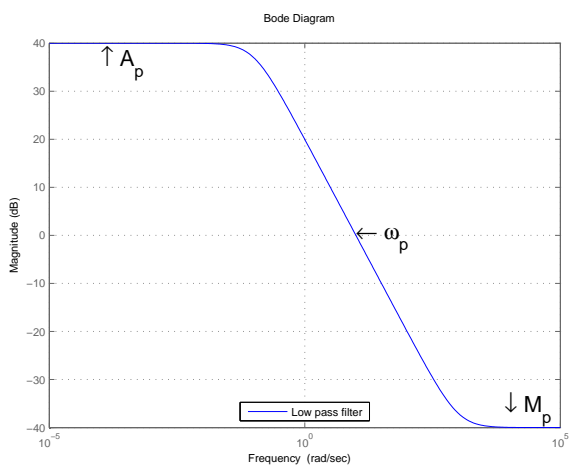

Fig. 4. Air gap weighting filter structure.

\section{NSGAII implementation}

The parameters used are shown in Table III. The crossover probability is generally selected to be large in order to have a good mixing of genetic material. The mutation probability is defined as $1 / n_{v}$, where $n_{v}$ is the number of variables. This is appropriate in order to give a mutation probability that mutates an average of one parameter from each individual. Mutation parameter changes dynamically during the optimisation as it depends on the number of sensor sets used (for each sensor a scalar weighting function is assigned which requires one variable each). For the simulated binary crossover parameter (SBX) and the mutations parameter it was decided to use the default value of 10 and 50 since they provide good distribution of solutions for the algorithm operations. The number of population is set as 50 and the generations number is been set to depend on the sensor set size. The total number of variables is eight using the full sensor set. The generation number $\left(G_{e n} n_{\text {num }}\right)$ is set to 150 for sensor sets with 1,2 or 3 sensors. For more sensors in a set, Gen num $_{\text {m }}=200$ (however this can be a designer choice as it can reduce or increase time consumption). To achieve the required constraints, different ways exist in genetic algorithms [1]. The penalty function approach [3] is 
TABLE III

NSGA-II PARAMETERS USED FOR THE EVOLUTION PROCEDURE.

\begin{tabular}{|c|c|}
\hline Parameter & setting \\
\hline Crossover probability & 0.9 \\
Mutation probability & $1 / n_{u}$ \\
SBX parameter & 10 \\
Mutation parameter & 50 \\
Rigid bounds & 1 (yes) \\
Population & 50 \\
Generations & Gen $_{n u m}$ \\
\hline
\end{tabular}

used to achieve the constraint within limits. The constraint violation for each constraint, $k^{i}$, defined in Table II, is given as

$$
\omega_{j}\left(k^{j}\right)= \begin{cases}\left|g_{j}\left(k^{j}\right)\right|, & \text { if } g_{j}\left(k^{j}\right)<0 \\ 0 & \text { otherwise }\end{cases}
$$

Each soft constraint is normalised as in (19) for values less than the predefined level $g_{j l o w}$ is used and $g_{j h i g h}$ is used for values higher than the predefined.

$$
g_{\text {jlow }}=-\frac{k^{j}}{k_{\text {des }}^{j}}+1 \geq 0 \quad g_{\text {jhigh }}=\frac{k^{j}}{k_{\text {des }}^{j}}-1 \geq 0
$$

Where, $k_{\text {des }}^{j}$ is the predefined constraint value and $k^{j}$ is the measured value. The hard constraint violation is given as

$$
\psi_{i}\left(f^{i}\right)= \begin{cases}0, & \text { if } h_{i}\left(f^{i}\right)=0 \\ \left|h_{i}\left(f^{i}\right)\right| & \text { otherwise }\end{cases}
$$

This is transformed into a soft constraint, allowing a small tolerance value $\sigma$. Therefore, the steady state error for the airgap is given as

$$
h_{i}=\left|f^{i}\right|-\sigma<0
$$

Where $f^{i}$ is the steady state error of the control effort that eventually controls the steady state error for the airgap.

The overall constraint violation is given in (22) is to be used as a metric for the controllers performance towards the given constraints.

$$
\Omega\left(k^{(j)}, f^{(i)}\right)=\sum_{j=1}^{j} \omega_{j}\left(k^{(i)}\right)+\sum_{i=1}^{i} \psi_{i}\left(f^{(i)}\right)
$$

This constraint violation is then added to each of the objective functions values

$$
\Phi_{m}=\phi_{m}+R_{m} \Omega\left(k^{(i)}, f^{(i)}\right)
$$

where $R_{m}$ is the penalty parameter and $\Phi_{m}$ the objective function value. In this case, a dynamically updated penalty parameter is required. This is useful, in order to avoid infeasible solutions and the penalty parameter is set to be a function of the generation number [10]. The penalty parameters are finalized as follows:

$$
R_{i_{r m s}}=C * 1, \quad R_{\ddot{z}_{r m s}}=C * 0.5, \quad R_{\gamma_{o p t}}=C * 1
$$

With, $\mathrm{C}$ being the generation number for the current sensor set.

\section{Simulations AND DATA ANAlysis}

The flow chart of the framework is depicted in Fig. 5, showing how NSGAII is merged to the sensor selection framework efficiently, producing sensor sets with the required criteria. First, the user defines the selection criteria for the controllers, i.e. ride quality, stability margin, RMS current or any other properties required based on the measurements (a total of 14 from stochastic and deterministic simulations). Then, one sensor set is selected and the optimisation commences with the NSGAII randomly generating parameters for the weighting functions. When the generations reach $G_{\text {enax }}$, the controllers that do not satisfy the constraints are rejected based on (22). Then the optimised controllers that satisfy the input criteria are selected and the algorithm proceeds to the next sensor set until the overall optimisation is over. The overall algorithm was tested using a Pentium 4 , Dual core processor running at $2 \mathrm{GHz}$ with $4 \mathrm{~GB}$ DDR memory and without the Java tool of MATLAB 7.2. The average simulation time per sensor set with all tasks is about 1.5 hours. The optimisation for all possible sensor sets required approximately 26 hours resulting in a total of 800 optimised controllers to choose from (at this stage the simulations run on the nominal system). The results show

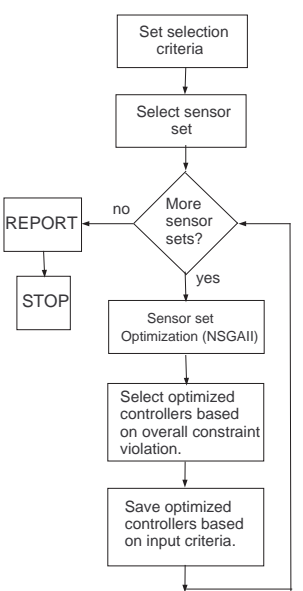

Fig. 5. Sensor optimisation flow chart.

that all sensor sets meet the assigned constraints including the relaxations. A sample from the results obtained is shown in Table IV. Column $\Omega$ indicates the overall constraint violation that is satisfied for all sensor sets. Column $n[K(s)]$ is the number of controllers that satisfy the input criteria from each sensor set. To illustrate, we introduce the following input criteria:

$$
\gamma<4 \quad \ddot{z}<0.5 \mathrm{~m} / \mathrm{s}^{2}
$$

Those two criteria ensure that the selected optimised controllers will result in a MAGLEV performance that is working within the normal constraints mentioned in Table II. With the proposed filters, i.e the simplified filter structures, with only the primary airgap filter being dynamic, the airgap sensor and any double sensor combination do not satisfy the strict robust stability radius and ride quality in (25). However, 
TABLE IV

SENSOR COMBINATIONS RELATING TO CRITERIA IN (25)

\begin{tabular}{|c|c|c|c|}
\hline & Sensor set & $\Omega$ & $n[K(s)]$ \\
\hline 1 & $\left(z_{t}-z\right)$ & $\sqrt{ }$ & 0 \\
2 & $\left(z_{t}-z\right), \dot{z}$ & $\sqrt{ }$ & 0 \\
3 & $\left(z_{t}-z\right), \ddot{z}$ & $\sqrt{ }$ & 0 \\
4 & $\mathrm{i}, \mathrm{b},\left(z_{t}-z\right)$ & $\sqrt{ }$ & 32 \\
5 & $\mathrm{i}, \mathrm{b},\left(z_{t}-z\right), \ddot{z}$ & $\sqrt{ }$ & 35 \\
6 & $\mathrm{i},\left(z_{t}-z\right), \dot{z}, \ddot{z}$ & $\sqrt{ }$ & 41 \\
7 & $\mathrm{~b},\left(z_{t}-z\right), \dot{z}, \ddot{z}$ & $\sqrt{ }$ & 50 \\
8 & $\mathrm{i}, \mathrm{b},\left(z_{t}-z\right), \dot{z}, \ddot{z}$ & $\sqrt{ }$ & 50 \\
\hline
\end{tabular}

they do satisfy the related criteria as set in the framework originally. This is expected because with simple filter structures, i.e to illustrate the concept rather than introducing complexity at this stage, more sensor information is needed. This can be clearly seen in the three sensor measurement sets that provide feasible controllers. However, any single or double measurements could be used for a fault tolerant framework under given system requirements. In particular, these could be utilised in the Fault tolerant structure for graceful degradation of performance before reconfiguration.

The $i, b,\left(z_{t}-z\right)$ sensor set pareto optimum consisting of 50 optimised controllers is shown in Fig. 6. It can be seen that the robust stability margin $\epsilon$ is within 0.25 and the vertical acceleration is less than $1 \mathrm{~m} / \mathrm{s}^{2}$ and in many cases, less that $0.5 \mathrm{~m} / \mathrm{s}^{2}$. The trade-off between the ride quality and the RMS current is also shown although is rather difficult to see the figure due to the 3D nature. Fig. 7 illustrates the

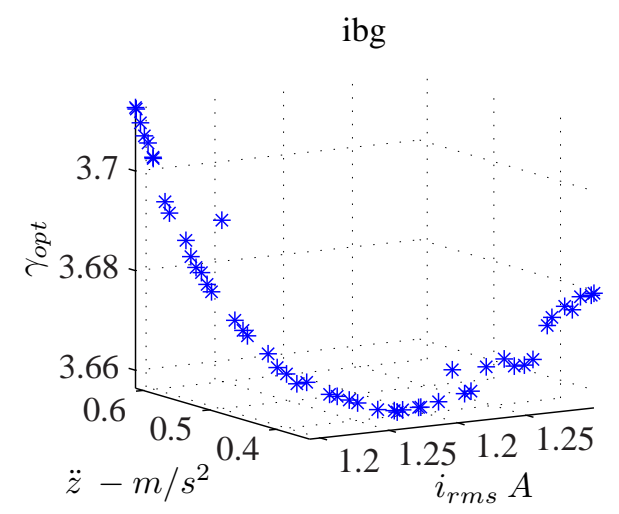

Fig. 6. Pareto front with $i, b,\left(z_{t}-z\right)$ measurement set.

deterministic airgap for each optimised controller and the corresponding control effort for the $i, b,\left(z_{t}-z\right)$ sensor set. Clearly, the airgap deflection is within the required limits with the settling time constraint satisfied. The corresponding control effort follows a similar behaviour settling at a peak value of about $50 \mathrm{~V}$. The pareto front of the optimised controllers from the sensor set $i, b,\left(z_{t}-z\right), \ddot{z}$ is depicted in Fig. 8. From the plot, it can be seen that the pareto front is successfully recovered from the NSGAII. Its clear that all optimised controllers meet the required robustness criteria with a ride quality lying within the preset limits. The airgap deflection for the sensor set as well as the resulting control

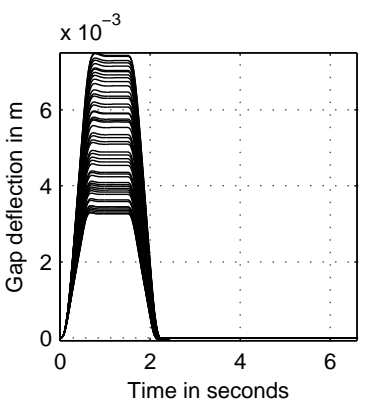

(a) Air gap deflection.

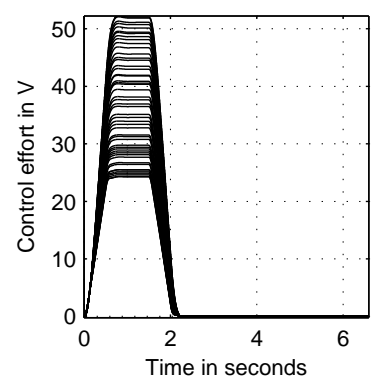

(b) Control effort.
Fig. 7. Air gap deflections and control effort with i,b, $\left(z_{t}-z\right)$.

effort from the optimised controllers is shown in Fig. 9. The maximum airgap deflection is within limits and both the settling time and steady state error are satisfied. The control effort reaches a maximum peak value of about $50 \mathrm{~V}$. This indicates, that regardless the fact that more information is used ( 4 sensors) the optimised controllers do not change the performance significantly compared to that of the previous case of three sensors (i.e. three sensor could be implemented rather than four).

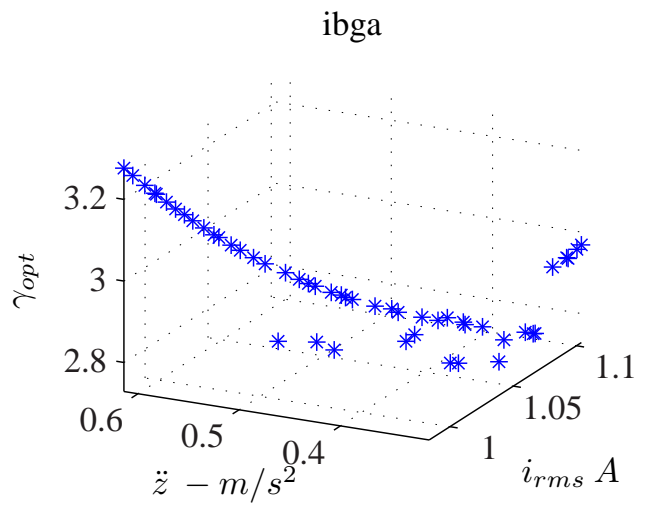

Fig. 8. Pareto front with $i, b,\left(z_{t}-z\right), \ddot{z}$ sensor set.

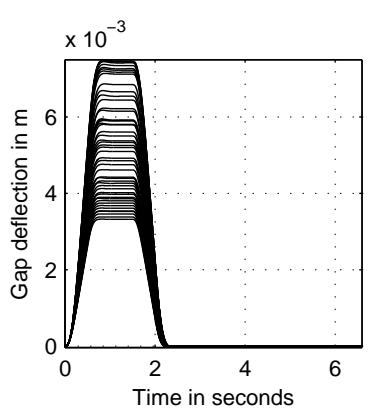

(a) Air gap deflection.

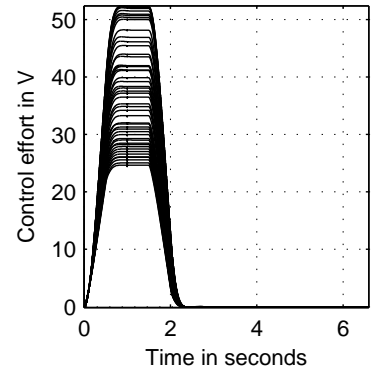

(b) Control effort.
Fig. 9. Air gap deflections and control effort with $i, b,\left(z_{t}-z\right), \ddot{z}$ set.

\section{ROBUSTNESS ANALYSIS}

In this section, the parametric uncertainty for the MAGLEV suspension is considered for robust stability and 
robust performance of the optimised controllers. The parametric nominal values of the MAGLEV are varied as shown in Table I. Note that the LSDP design procedure considers the model uncertainty in the coprime factor description. In this section, we consider the performance of the scheme under parametric uncertainty and how this could be extended for future work perhaps including fault tolerance system. Thus, a randomly picked-up optimised controller is selected from each sensor set in order to investigate the effect of parametric uncertainty on stability and performance. The stability is found from the eigenvalues and the performance to parametric uncertainties is reflected on the constraint violation. For example, if the nominal controller selected has an overall constraint $\Omega_{n}$, then the model perturbations, will result in a range of overall constraints values that have minimum and maximum values $\left(\Omega_{\min }, \Omega_{\max }\right)$. Therefore, 20 perturbed samples will be noted to check for robustness. The results are presented in Table V. All optimised sensor configurations are stable under the tested perturbed plants. 11

TABLE V

ROBUSTNESS FOR SENSOR CONFIGURATIONS.

\begin{tabular}{|c|c|c|c|c|c|}
\hline id & Sensor set & $\Omega_{n}$ & $\Omega_{\min }$ & $\Omega_{\max }$ & stable \\
\hline 1 & $\left(z_{t}-z\right)$ & 1.26 & 1.2 & $8 \times 10^{8}$ & $\sqrt{ }$ \\
2 & $\left(z_{t}-z\right), \dot{z}$ & 1.22 & 0.68 & 8.99 & $\sqrt{ }$ \\
3 & $\mathrm{i},\left(z_{t}-z\right), \ddot{z}$ & 0.14 & 0.14 & 0.14 & $\sqrt{ }$ \\
4 & $\mathrm{i}, \mathrm{b},\left(z_{t}-z\right)$ & 3.65 & 0 & 0.08 & $\sqrt{ }$ \\
5 & $\mathrm{~b},\left(z_{t}-z\right), \ddot{z}$ & 0 & 0 & 0 & $\sqrt{ }$ \\
6 & $\mathrm{i}, \mathrm{b},\left(z_{t}-z\right), \ddot{z}$ & 0 & 0 & 0 & $\sqrt{ }$ \\
7 & $\mathrm{~b},\left(z_{t}-z\right), \dot{z}, \ddot{z}$ & 0 & 0 & 0 & $\sqrt{ }$ \\
8 & $\mathrm{i}, \mathrm{b},\left(z_{t}-z\right), \dot{z}, \ddot{z}$ & 0 & 0 & 0 & $\sqrt{ }$ \\
\hline
\end{tabular}

out of 16 sensor sets do not satisfy, the robust performance as the overall constraint changes in some cases significantly, e.g. in sensor set id:1. In some cases as in sensor set id:2, the performance does not change significantly therefore it could be used, for a fault tolerant controller scheme. This is expected, as the optimised configurations are designed under the relaxed condition of the $\epsilon>0.15$. Sensor sets id: 3,4,5,6,7,8 perform rather well, as the nominal constraint violation remains constant. The perturbed airgap deflections of sensor sets id:4 and id:6 is depicted in Fig. 10. It can be seen that the airgap deflection remains almost unchanged under parametric changes for sensor set id:6; slightly pertubed responses appear in the smaller sensor set id:4 but within the required constraint. Hence, the overall constraint violation value could be used to indicate the performance of the closed loop response and could be used in the optimisation framework to select a robust performance response.

\section{CONCLUSIONS AND FUTURE WORK}

In this paper, a framework for the sensor optimisation for a MAGLEV suspension is presented. MacFarlane's and Glover's $H_{\infty}$ loop-shaping design along with the genetic algorithm perfoms well, producing optimised controllers for each sensor set that can satisfy the designer's predefined limitations. Robustness on parametric uncertainties was also investigated with the controller performing appropriately.
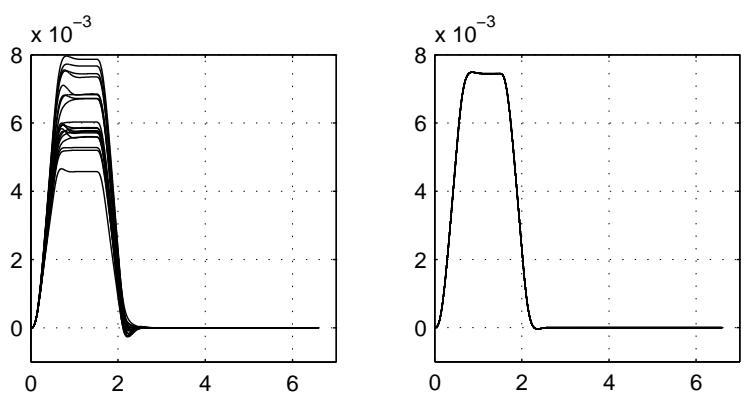

(a) Perturbed Air gap deflection (b) Perturbed Air gap deflection with $\mathbf{i}, \boldsymbol{b},\left(z_{t}-z\right)$ sensor set. $\quad$ with $\mathbf{i}, \boldsymbol{b},\left(z_{t}-z\right), \ddot{z}$ sensor set.

Fig. 10. Air gap deflections with 20 perturbed samples using 2 sensor sets.

The overall procedure could assist towards the development of a fault tolerance control system that is able to utilize bank of controllers and replace the appropriate ones depending on the sensor fault. Thus, keeping the suspension running within appropriate performance limits. Current work is considering dynamic filters for all measurements, aiming to reduce the sensor number in a set and quantify the resulting complexity of the overall controllers.

\section{REFERENCES}

[1] Coello C.A.C. Theoretical and numerical constraint-handling techniques used with evolutionary algorithms: A survey of the state of the art. Computer Methods in Applied Mechanics and Engineering, 191(11-12):1245-1287, 2002.

[2] N. V. Dakev, J. F. Whidborne, A. J. Chipperfield, and P. J. Flemings. Evolutionary $\mathrm{h}$ infinity design of an electromagnetic suspension control system for a maglev vehicle. Proceedings of the Institution of Mechanical Engineers.Part I, Journal of Systems \&amp; Control Engineering, 211(5):345-355, 1997.

[3] Kalyanmoy Deb. Multi-objective Optimization using Evolutionary Algorithms. John Wiley \&amp; sons Ltd, 2001.

[4] Kalyanmoy Deb, Amrit Pratap, Sameer Agarwal, and T. Meyarivan. A fast and elitist multiobjective genetic algorithm: Nsga-ii. IEEE Transactions on Evolutionary Computation, 6(2):182-197, 2002.

[5] J. Dreo, P. Siarry, A. Petrowski, and E. Taillard. Metaheuritics for Hard Optimization. Springer-Verlg Berlin Heidelberg, New York, 2006.

[6] P. J. Fleming and R. C. Purshouse. Evolutionary algorithms in control systems engineering: A survey. Control Engineering Practice, 10(11):1223-1241, 2002.

[7] R. M. Goodall. Dynamic characteristics in the design of maglev suspensions. Proceedings of the Institution of Mechanical Engineers, Part F: Journal of Rail and Rapid Transit, 208(1):33-41, 1994.

[8] R. M. Goodall. Dynamics and control requirements for ems maglev suspensions. In Proceedings on international conference on Maglev, pages 926-934, Oct 2004.

[9] R. M. Goodall. The theory of electromagnetic levitation. Physics in Technology, Vol. 16(No 5):pp 207-213, Sept 1985.

[10] Jeffrey A. Joines and Christopher R. Houck. On the use of nonstationary penalty functions to solve nonlinear constrained optimization problems with ga's. Proceedings of the IEEE Conference on Evolutionary Computation, ICEC, 1994.

[11] Abdullah Konak, David W. Coit, and Alice E. Smith. Multi-objective optimization using genetic algorithms: A tutorial. Reliability Engineering and System Safety, 91(9):992-1007, 2006.

[12] Hyung-Woo Lee, Ki-Chan Kim, and Ju Lee. Review of maglev train technologies. IEEE Transactions on Magnetics, 42(7):1917-1925, 2006.

[13] Duncan McFarlane and Keith Glover. A loop-shaping design procedure using $\mathrm{h}$ infinity synthesis. IEEE Transactions on Automatic Control, 37(6):759-769, 1992.

[14] S. Skogestad and I. Postlethwaite. Multivariable Feedback Control Analysis and Design. John Wiley \&amp; Sons,Ltd, 2005. 